\title{
Use of contrast-enhanced computed tomographic imaging to diagnose and evaluate Behçet's disease with vascular complications
}

\author{
LIN QI $^{1 *}$, JIANFEI CAI $^{2 *}$, DINGBIAO MAO ${ }^{1 *}$, MING WANG $^{1}$, XIAOJUN GE $^{1}$, \\ WEILAN WU ${ }^{1}$, XIU JIN ${ }^{1}$, CHENG LI ${ }^{1}$, YANQING HUA ${ }^{1}$ and MING LI ${ }^{1,3}$ \\ Departments of ${ }^{1}$ Radiology and ${ }^{2}$ Rheumatology, Huadong Hospital Affiliated to Fudan University, Shanghai 200040; \\ ${ }^{3}$ Institute of Functional and Molecular Medical Imaging, Fudan University, Shanghai 201318, P.R. China
}

Received March 21, 2019; Accepted September 4, 2019

DOI: $10.3892 /$ etm.2019.8088

\begin{abstract}
In the present study, multi-slice $\mathrm{CT}$ results of patients with Behçet's disease (BD) and vascular complications were retrospectively evaluated. From January 2016 to May 2018, 45 of 361 patients with BD were diagnosed with vascular involvement. The clinical background, laboratory parameters and response to therapy of those patients were assessed. The following characteristics of vascular aneurysms were analyzed: Maximum diameter, length, wall thickness, borders, luminal changes, mural thrombus, cystic change of the vessel walls, asymmetric bulging of the right part of the aortic wall (RP type) and calcific plaques. The 45 BD patients analyzed included 37 males and 8 females with a median age of 40 years (30-49 years). Significant differences were observed among genders regarding age, ocular disorders and digestive-tract ulceration. A total of 42 aneurysms were identified with a mean diameter of $43 \mathrm{~mm}$. Most aneurysmal walls (88\%) were homogeneously enhanced on contrast-enhanced CT. Comparison of groups classified by aortic and larger arterial aneurysms indicated that aneurysms occurring in the aorta were more likely to form a mural thrombus, have a thicker wall $(\mathrm{P}<0.001)$ and unclear borders $(\mathrm{P}=0.036)$, to be of the RP type $(\mathrm{P}=0.003)$ and have a longer extension $(\mathrm{P}=0.001)$ compared with those in larger arteries. Unclear border of the aneurysmal wall was the only radiologic predictor correlated with an elevated erythrocyte sedimentation rate $(\mathrm{P}<0.001)$. In conclusion, characteristic CT imaging features of aneurysms
\end{abstract}

Correspondence to: Dr Ming Li or Dr Yanqing Hua, Department of Radiology, Huadong Hospital Affiliated to Fudan University, 221 West Yan'an West Road, Building 8, 4th Floor, Shanghai 200040, P.R. China

E-mail: minli77@163.com

E-mail: doc.yanqinghua@126.com

*Contributed equally

Key words: Behçet's disease, aneurysm, vasculitis, computed tomography, corticosteroids may help to diagnose vascular involvement of $\mathrm{BD}$ and assess its severity, particularly in the absence of the classical clinical manifestations.

\section{Introduction}

Behçet's disease (BD) is a chronic and multi-systemic autoimmune disorder of unknown pathogenesis that is associated with relapsing oral and genital ulcerations, ocular manifestations and additional clinical manifestations in multiple organ systems, including the skin, joints, central nervous system, gastrointestinal tract and lungs. BD mainly occurs in young adults between 20 and 40 years of age and is more common in males (1). Vascular involvement with underlying pathologic processes of vasculitis and perivascular inflammatory infiltrates develops in 20-40\% of patients with BD and is considered the most serious complication, with a mortality rate of $60 \%$ in patients with aortic aneurysm formation (2-4). Arterial complications are reported to develop within 3.5-25 years after the diagnosis of BD (5); thus, early detection of vascular abnormalities is imperative to prolong the survival of BD patients with vascular complications. CT has an important role in diagnosing and assessing complications involving different organs and systems. The present study provided a summary of notable cardiovascular complications of $\mathrm{BD}$ focusing on the characteristic imaging manifestations that should be noted by clinicians and radiologists.

\section{Materials and methods}

Case selection. From January 2016 to May 2018, 361 patients were diagnosed with BD at the Huadong Hospital affiliated to Fudan University (Shanghai, China) based on diagnostic criteria suggested by the International Study Group for BD (6). Due to lack of characteristic laboratory and histopathologic findings, $\mathrm{BD}$ is diagnosed based on clinical symptoms. The diagnostic criteria for BD include relapsing oral ulceration plus 2 of the following: Recurrent genital ulceration, ocular involvement, skin lesions and a positive pathergy test. Patients with cancer, rheumatoid arthritis, diabetes and syphilis were excluded. The patients underwent chest and abdominal contrast-enhanced 
CT examination (100\%), upper or lower extremity vascular ultrasound (27.1\%), MR angiography (23.5\%) and cardiac color Doppler ultrasound (100\%). Among them, 45 patients $(12.4 \%)$ had confirmed vascular involvement as determined by CT angiography $(21,91.3 \%)$, lower extremity vascular ultrasound $(2,8.7 \%)$ and MR angiography $(1,4.3 \%)$. The patients' clinical background, laboratory results, $\mathrm{CT}$ images and response to therapy (range of follow-up time, 3-42 months) were assessed and analyzed. The outcome endpoints during follow-up were death due to aneurysmal rupture, severe hemoptysis and recurrence after surgery, including post-operative perivalvular leakage, progression of aneurysm and restenosis of the in-stent coronary artery. This study was conducted retrospectively at one center, where the local ethical institutional review board of Huadong Hospital (Shanghai, China) waived the need for informed consent for this study.

Radiological examination. CT angiography was performed on the 45 patients with a 64-slice multi-slice (MS)CT scanner (Sensation 64; Siemens Medical Solutions). The scanning parameters were as follows: Section thickness, $2.5 \mathrm{~mm}$; tube voltage, $120 \mathrm{kVp}$; and tube current, $300 \mathrm{~mA}$. A dual-source power injector (CT motion; Ulrich Medical) was used for all examinations. After placing an $18-\mathrm{G}$ intravenous catheter through the right antecubital vein for all patients, $80-100 \mathrm{ml}$ of the contrast medium iopromide (370 mg iodine $/ \mathrm{ml}$; Bracco) was injected at a flow rate of 4.5-5.5 ml/sec based on the body mass index and vein condition, followed by a $30-$ or $40-\mathrm{ml}$ saline chaser at the same flow rate. A region of interest was placed in the ascending thoracic aorta and image acquisition was automatically initiated once a selected threshold (120 Hounsfield units) had been reached with bolus tracking. The venous phase was obtained with a delay of between 90 and $120 \mathrm{sec}$ after injection. The patients' clinical background, laboratory results, CT images and response to therapy were assessed and analyzed.

Imaging analysis. Images from all of the 45 patients were analyzed by two radiologists (LQ and DBM) each with 7 years of diagnostic experience using CT angiography, and their decisions were reached by consensus. The location and number of aneurysms and other types of lesions, including wall thickening without aneurysm formation and thrombosis of the aorta, were measured and recorded for all cases. The following characteristics of vascular aneurysms were analyzed: Maximum diameter, length, wall thickness, border (clear or unclear), luminal changes (dilated or normal), mural thrombus (present or absent), cystic changes of the vessel wall (present or absent), asymmetric bulging of the right part of the aortic wall (RP-type) or uniform dilation, and calcific plaques (slight, obvious or not significant).

Statistical analysis. Statistical analysis was performed using SPSS 22.0 software (IBM Corp.) and GraphPad Prism (version 8.0; Graph-Pad Software, Inc.). Values were expressed as either the mean \pm standard deviation or median with interquartile range after testing the normality of variables using Shapiro-Wilk test. Subsequently, data were compared between different groups by using Pearson's chi-squared test for categorical variables, independent-samples t-test for continuous variables with a normal distribution, and a Mann-Whitney U-test for continuous variables with a skewed distribution. Kaplan-Meier survival curves and log-rank test were used for the survival outcomes of groups with and without aortic and larger arterial aneurysms. Bivariate correlation tests were performed to determine the dependence of the MSCT features of the aneurysms and the ESR. $\mathrm{P}<0.05$ was considered to indicate a statistically significant difference.

\section{Results}

Clinical presentation. The $45 \mathrm{BD}$ patients with vascular lesions included 37 males and 8 females (Table I). The median age was 40 years (total range, 25-73 years), The median course of BD was 8 years (total range, 1-40 years), including multiple pulmonary aneurysms found in a 30-year-old patient without previous history of BD (Fig. 1A and B). The median time to detect vascular complications after onset of BD was 3 years (total range, 0-40 years). Vasculitis-associated symptoms (Table SI) included chest tightness and shortness of breath $(22,48.9 \%)$, abdominal pain $(14,31.1 \%)$, backache $(4,8.9 \%)$, left lower extremity pain $(8,17.8 \%)$ and hoarseness $(3,6.7 \%)$. Certain patients did not have any symptoms associated with vasculitis $(10,22.2 \%)$. One patient had a recurrent fever with cough, sputum and relapsing hemoptysis. Among the 45 patients, $44(97.8 \%)$ had an elevated erythrocyte sedimentation rate (ESR) during hospitalization, 22 patients (48.9\%) had elevated C-reactive protein (CRP) levels, white blood cell counts and D-dimer levels, 16 patients $(35.6 \%)$ had elevated neutrophil counts, and $14(31.1 \%)$ and 6 patients $(13.3 \%)$ had decreased hemoglobin levels and platelet counts, respectively.

$B D$ at other sites. Except for vascular lesions, manifestations of BD in other organs, including relapsing oral ulceration (100\%), recurrent genital ulceration $(n=31,66 \%)$, skin lesions including epifolliculitis and erythema $(n=29,64.4 \%)$, ocular $(n=9,20 \%)$ and joint $(n=4,8.9 \%)$ involvement, digestive ulceration $(n=7$, $15.6 \%$ ) and heart abnormalities (right ventricular thrombosis and endocarditis; $n=8,17.8 \%$ ) are listed in Table II. One patient had central nervous system involvement with symptoms including a sudden inability to speak, limb weakness and a right pupil unresponsive to light. A brain MR examination indicated multiple abnormal signals in the right frontal lobe, temporal lobe, bilateral occipital cortex and brainstem.

Significant differences were observed between genders regarding age, ocular disorders, blood CRP and neutrophil levels $(\mathrm{P}<0.05)$, as well as digestive ulceration $(\mathrm{P}<0.01)$. No sex difference was observed regarding the onset age of $\mathrm{BD}$ and the onset of vascular complications, incidence rate of organic disorders other than those of the eyes and digestive tract, or the leukocyte count.

Vascular aneurysms. A total of 42 aneurysms were identified in 38 patients. Comparison of CT features and laboratory data between two groups with either aortic or larger artery aneurysms is provided in Table II. A total of 14 patients $(14 / 45,31.1 \%)$ had multiple vascular lesions, and 10 patients had aneurysm and thrombosis $(22.2 \%)$. A total of 28 aneurysms were located in the aorta $(28 / 42,66.7 \%)$ including 20 lesions in the thoracic aorta $(20 / 28,71.4 \%)$ and 8 in the 
Table I. Clinical characteristics and laboratory parameters of the patients by gender.

\begin{tabular}{|c|c|c|c|c|c|}
\hline Item & Total & Males & Females & Healthy range & P-value \\
\hline Patients & 45 & $37(82.2)$ & $8(17.8)$ & - & 0.012 \\
\hline Age (years) & $40(25-73)$ & $40(25-73)$ & $40(30-70)$ & - & 0.037 \\
\hline Onset age of BD (years) & $11.7 \pm 11.4$ & $30.7 \pm 9.0$ & $28.5 \pm 10.3$ & - & 0.085 \\
\hline $\begin{array}{l}\text { Detection time of vascular complications } \\
\text { after BD (years) }\end{array}$ & $3(0-40)$ & $3(0-40)$ & $4.5(3-37)$ & - & 0.113 \\
\hline Duration of BD (years) & $8(1-40)$ & $6.0(1-40)$ & $22.3 \pm 19.2$ & - & 0.223 \\
\hline Clinical presentation & & & & - & \\
\hline Oral ulcerations & $45(100)$ & 37 (100) & $8(100)$ & - & 0.408 \\
\hline Genital ulcerations & $31(66.0)$ & $25(67.6)$ & $6(75)$ & - & 0.681 \\
\hline Skin lesions & $29(64.4)$ & $25(67.6)$ & $4(50)$ & - & 0.347 \\
\hline Arthritis & $4(8.9)$ & $2(5.4)$ & $2(10)$ & - & 0.077 \\
\hline Ocular disorders & $9(20)$ & $5(13.5)$ & $4(50)$ & - & 0.019 \\
\hline Fever & $1(2.2)$ & $1(2.7)$ & n.d. & - & n.d. \\
\hline Digestive ulceration & $7(15.6)$ & $3(8.1)$ & $4(50)$ & - & 0.003 \\
\hline Heart disorders & $8(17.8)$ & $8(21.6)$ & 0 & - & 0 \\
\hline Laboratory examination & & & & - & \\
\hline $\operatorname{ESR}(\mathrm{mm} / \mathrm{h})$ & $15(4-89)$ & $7(4-89)$ & $16.0(5.0-60)$ & $0-15 / 0-20$ & 0.827 \\
\hline CRP (mg/l) & $8.8(1.2-117)$ & $10.3(1.2-117)$ & $3.75(4.8-8.3)$ & $<10$ & 0.001 \\
\hline Leukocytes $\left(10^{9} / 1\right)$ & $8.5(4.6-12.4)$ & $10.80(4.70-12.4)$ & $6.5 \pm 1.4$ & $4-10$ & 0.088 \\
\hline Neutrophils (\%) & $58.1(40.2-82.6)$ & $58.8(47.3-82.6)$ & $51.6 \pm 9.4$ & $50-70$ & 0.011 \\
\hline Hemoglobin $(\mathrm{g} / \mathrm{l})$ & $135(24.6-156)$ & $135(24.6-156.0)$ & $134.8 \pm 10.4$ & $110-160$ & 0.651 \\
\hline Thrombocytes $\left(10^{9} / 1\right)$ & $176(106.0-393.0)$ & $172.0(106.0-393.0)$ & $184.3 \pm 6.6$ & $100-300$ & 0.161 \\
\hline D-Dimer elevation & $22(48.9)$ & $20(54.1)$ & $2(5.3)$ & $<0.2$ & 0.136 \\
\hline
\end{tabular}

Values are expressed as the mean \pm standard deviation for normally distributed data and as the median with total range for data with a skewed distribution. Normal level: ESR, 0-15 mm/h for male adult, 0-20 mm/h for female adult; CRP, <10 mg/l; leukocytes, 4-10x10 $/ 1$; neutrophils, 50-70\%; hemoglobin, 110-160 g/l; thrombocytes, 100-300x10 $/ 1$; D-dimer elevation, $<0.2 \mathrm{mg} / \mathrm{l}$. Years of vasculitis after BD: Time to develop vascular complications after the onset of BD. BD, Behçet's disease; ESR, erythrocyte sedimentation rate; CRP, C-reactive protein.

abdominal aorta $(8 / 28$ cases, $28.6 \%)$. Among the patients with abdominal aortic aneurysms, two were found with recurrent saccular pseudoaneurysm by CT examination (Fig. 1C and D). Furthermore, 14 aneurysms were located in large arteries $(14 / 42,33.3 \%)$, including the right brachiocephalic trunk $(2 / 14,14.3 \%)$, right internal thoracic artery $(1 / 14,7.1 \%)$, right carotid artery $(2 / 14,14.3 \%)$, left femoral artery $(2 / 14,14.3 \%)$, iliac arteries $(5 / 14,35.7 \%)$ and right pulmonary artery $(2 / 14$, $14.3 \%)$. Of the 20 aneurysms in the thoracic aorta, $18(90 \%)$ were located in the aortic sinus, ascending aorta and aortic arch, and $2(10 \%)$ were located in the descending aorta. Of the 8 aneurysms in the abdominal aorta, 6 lesions were located in the suprarenal abdominal aorta and 2 were located in the infrarenal abdominal aorta. Of all 42 aneurysms, 14 lesions $(33.3 \%)$ were located below the diaphragm and 28 lesions (66.7\%) were located above the diaphragm.

The diameter of the 42 aneurysms ranged from 13 to $118 \mathrm{~mm}$ (mean, $43 \mathrm{~mm}$ ), and the length ranged from 7 to $130 \mathrm{~mm}$ (mean, $43 \mathrm{~mm}$ ). The affected aortic or arterial walls were thickened in 34 aneurysms (81\%), with an average maximum thickness of $4.0 \mathrm{~mm}$ (range, 3-14 mm). Most aneurysmal walls $(30 / 34,88 \%)$ were homogeneously enhanced during the late phase of contrast-enhanced CT, while 4 of them
$(4 / 34,11.8 \%)$ had irregular cystic changes in the thickened walls. A total of 28 of the 42 aneurysms (66.7\%) had unclear borders and $14(33.3 \%)$ had a mural thrombus (Table II). In 10 aneurysms $(10 / 42,23.8 \%)$, atherosclerotic changes were present in the affected segments.

Furthermore, 20 aneurysms (20/42, 47.6\%) had asymmetric bulging of the right part of the aortic wall (RP-type). A large saccular pseudoaneurysm of RP-type was found in a patient with 13 years of BD history (Fig. 2A and B), whereas an asymmetric bulging of the aortic wall with mural thrombus formation was found another patient (Fig. 2C-E). A total of 14 aneurysms were located in the thoracic aorta $(14 / 20,70 \%)$, 5 were located in the abdominal aorta $(5 / 20,25 \%)$ and 1 was located in the left iliac artery $(10 \%)$. All of the 14 thoracic aortic RP-type aneurysms were located in the aortic sinus, ascending aorta and aortic arch. One aneurysm located in the left femoral artery manifested as asymmetric bulging of the left part of the arterial wall. In the remaining 20 aneurysms, the aortic or arterial walls were circumferentially enlarged.

Comparison between groups classified by aortic and larger arterial aneurysms (Table II) revealed that aneurysms occurring in the aorta were more likely to form a mural thrombus $(\mathrm{P}<0.001)$, have a thicker wall $(\mathrm{P}<0.001$, Fig. 3A) and unclear 

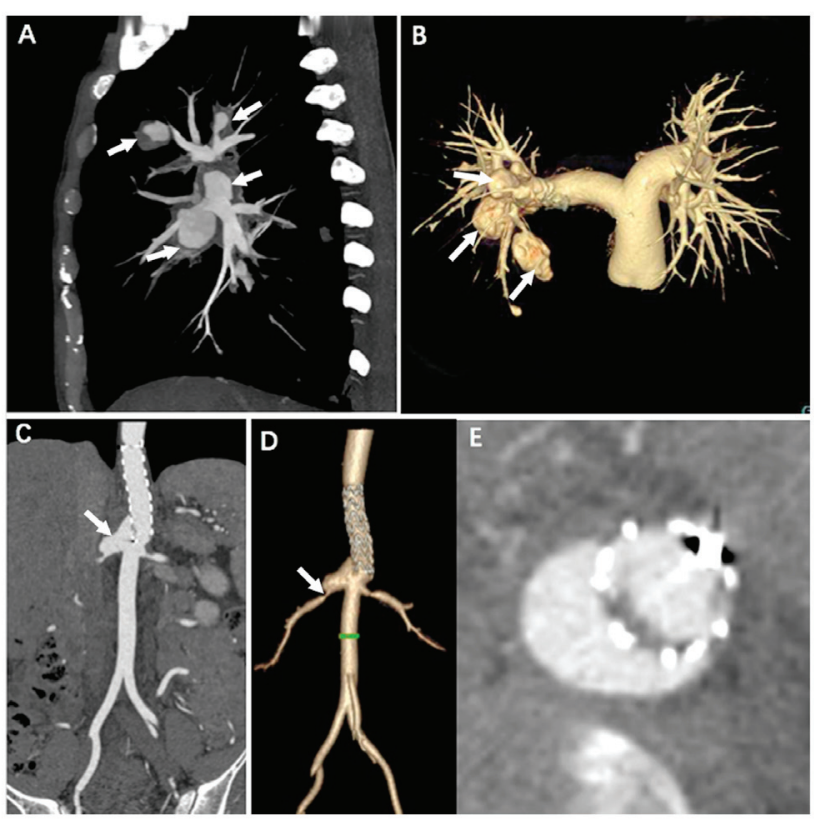

Figure 1. Patients with Behcet's disease (BD) with complications of pulmonary aneurysms and a recurrent saccular pseudoaneurysm after stent-graft implantation. (A and B) Multiple pulmonary artery aneurysms were found in a 30-year-old patient without known history of BD history for the symptom of hemoptysis. Multi-slice CT pulmonary angiographic examination was performed one day after hospitalization. (A) Sagittal reconstructed CT image indicating multiple pulmonary aneurysms (white arrow) visible as saccular dilations arising from the right pulmonary artery with mural thrombus formation. (B) Volume rendering image revealing multiple pulmonary aneurysms (white arrow) close to the hilus. (C-E) CT images 31 months after stent implantation. (C) Coronal reconstructed CT angiography indicating a recurrent saccular pseudoaneurysm (white arrow) at the distal end of the stent-graft. (D) Volume rendering image revealing a recurrent pseudoaneurysm involving the opening of the right renal artery (white arrow). The green line is a tracking mark of vessel diameter on the abdominal aorta. (E) Axial CT image displaying a recurrent aneurysm located in the right wall close to the distal aspect of the stent-graft. CT, computed tomography.

borders $(\mathrm{P}=0.036)$, to be of the $\mathrm{RP}$ type $(\mathrm{P}=0.003)$ and have a longer extension $(\mathrm{P}=0.001$, Fig. $3 \mathrm{~B})$ than those in larger arteries. No significant differences were observed in aneurysmal diameter (Fig. 3C), number of organic disorders, the presence of calcific plaques, as well as the ESR, CRP and leukocytes.

Bivariate correlation tests were performed to determine the dependence of the MSCT features of the aneurysms and the ESR. A correlation was identified between the borders of the aneurysmal wall and the ESR (correlation coefficient, 0.627; $\mathrm{P}<0.001$, Fig. 3D). However, the correlations between the blood ESR levels and the diameter (Fig. 3E), length, thickness, presence of calcific plaque and presence of mural thrombus of the aneurysmal walls were not significant.

Other types of vascular lesion. Of the 45 patients, 18 (40\%) had arterial thromboembolism. The majority of arteriovenous thromboemboli occurred in larger arteries, including the bilateral pulmonary arteries $(8 / 18,44.5 \%)$, bilateral renal arteries $(2 / 14,14.3 \%)$, superior mesenteric artery $(2 / 14,14.3 \%)$, right subclavian artery $(1,7.1 \%)$, subclavian artery $(3 / 14,21.4 \%)$ and coronary arteries $(2 / 14,14.3 \%)$. A total of 7 of the 45 patients had unilateral thrombosis of lower extremity veins (15.6\%);
2 had inferior vena cava thrombosis (4.5\%) and 2 had pulmonary artery thrombus (4.5\%). All of those patients with vein thrombosis had severe stenosis $(5 / 11,45.5 \%)$ and occlusion $(6 / 11,54.5 \%$; Table SI).

Response to treatment and follow-up. Of the 45 patients, 42 were treated with prednisone, cyclophosphamide and monoclonal anti-tumor necrosis factor (TNF) antibodies. Fig. 4A and B revealed an aneurysm located in the abdominal aorta without a thickened wall or mural thrombus formation, where no significant changes in the aneurysm were observed by ultrasound in the 6-month follow-up after medical treatment. Two patients refused medical treatment and one died after 9 months due to thoracic aortic rupture. A total of 9 patients had aneurysm shrinkage after medical therapy as determined by ultrasound. However, due to a lack of CT examinations during follow-up, changes in the vascular walls of those patients were not determined. In one case, diffuse occlusion of the stent and the adjacent lumen of the right coronary artery, which exhibited thickened vascular walls and unclear boundaries, were discovered by coronary CT angiography at a 20-month follow-up after coronary stent implantation (Fig. 4C and D). A total of 21 patients underwent surgical treatments, including endovascular graft exclusion $(n=4)$, Bentall surgery $(n=4)$, aortic valve replacement $(n=5)$, inferior vena cava filter placement $(n=3)$ and aneurysmectomy $(n=5)$.

The follow-up duration ranged from 3 to 42 months with a median follow-up period of 18 months. Kaplan-Meier survival curves for the survival outcomes of the groups with and without aortic and larger arterial aneurysms are provided in Fig. 3F. The outcome endpoints were death due to aneurysmal rupture $(\mathrm{n}=2)$, massive hemoptysis ( $>500 \mathrm{ml} / \mathrm{d}, \mathrm{n}=1)$, recurrence after surgery including post-operative perivalvular leakage $(n=4)$, progression of the aneurysm $(n=4)$ and restenosis of the in-stent coronary artery $(n=2)$. The two patients who died of aneurysmal rupture displayed irregular cystic changes in the aortic wall on reconstructed enhanced CT imaging. No overall significant difference was observed between the two survival curves (log-rank test, $\mathrm{P}=0.170$ ), but there was significant difference between the curves after 28 months of survival $(\mathrm{P}<0.01)$

\section{Discussion}

The most significant complications of BD are arterial involvement and aneurysm formation, which account for $12 \%$ of the vascular complications (7). The mortality rate associated with an untreated arterial aneurysm is reported to be up to $60 \%$ and arterial manifestations are reported to manifest 3.5-25 years after a diagnosis of BD (8). An initial precise diagnosis of vascular involvement due to BD is difficult, particularly in the absence of classical clinical manifestations, as it is a rare complication.

The present study provided several predictors for diagnosing and assessing the severity of vascular involvement associated with BD based on the results obtained; most patients with BD and vascular involvement were younger males, which is consistent with the results of other studies $(6,9,10)$. Serologically, an elevated ESR is a known predictive parameter associated with the inflammatory activity of aneurysms. In the present study, an 
Table II. Comparison of CT manifestations and laboratory data between groups of aortic and larger arterial aneurysms.

\begin{tabular}{|c|c|c|c|c|}
\hline Item & Total $(n=42)$ & Aorta $(n=28)$ & Larger arteries $(n=14)$ & P-value \\
\hline Diameter (mm) & $43.0(13-118)$ & $46.6 \pm 13.1$ & $31(13-67)$ & 0.208 \\
\hline Length (mm) & $43.0(7-130)$ & $44.5(32-130)$ & $26.0(7-56.0)$ & 0.001 \\
\hline Wall thickness (mm) & $4.0(3-14)$ & $4(3-14)$ & $3(1-4)$ & $<0.001$ \\
\hline Number of organic disorders & $4(3-4)$ & $4(3-4)$ & $4(3-4)$ & 0.683 \\
\hline RP type & $20(47.6)$ & $18(64.3)$ & $2(14.2)$ & 0.003 \\
\hline Unclear border & $28(66.7)$ & $22(78.6)$ & $6(42.9)$ & 0.036 \\
\hline Mural thrombus & $14(33.3)$ & $4(14.3)$ & $10(71.4)$ & $<0.001$ \\
\hline Calcific plaques & $8(19.0)$ & $4(14.3)$ & $4(28.6)$ & 0.357 \\
\hline $\operatorname{ESR}(\mathrm{mm} / \mathrm{h})$ & $18.0(4.0-89)$ & $16.5(5-89)$ & $20(4-75)$ & 0.228 \\
\hline CRP (mg/l) & $8.8(1.2-117)$ & $9.5(1.2-113.4)$ & $8.4(3.7-41.5)$ & 0.155 \\
\hline Leukocytes $\left(10^{9} / 1\right)$ & $10.8(4-17.3)$ & $10.2 \pm 4.6$ & $7.1(4.3-11.5)$ & 0.080 \\
\hline
\end{tabular}

Values are expressed as the mean \pm standard deviation for normally distributed data and as the median with total range for data with a skewed distribution. ESR, erythrocyte sedimentation rate; CRP, C-reactive protein; RP-type, asymmetric bulging of right part of the aortic wall.

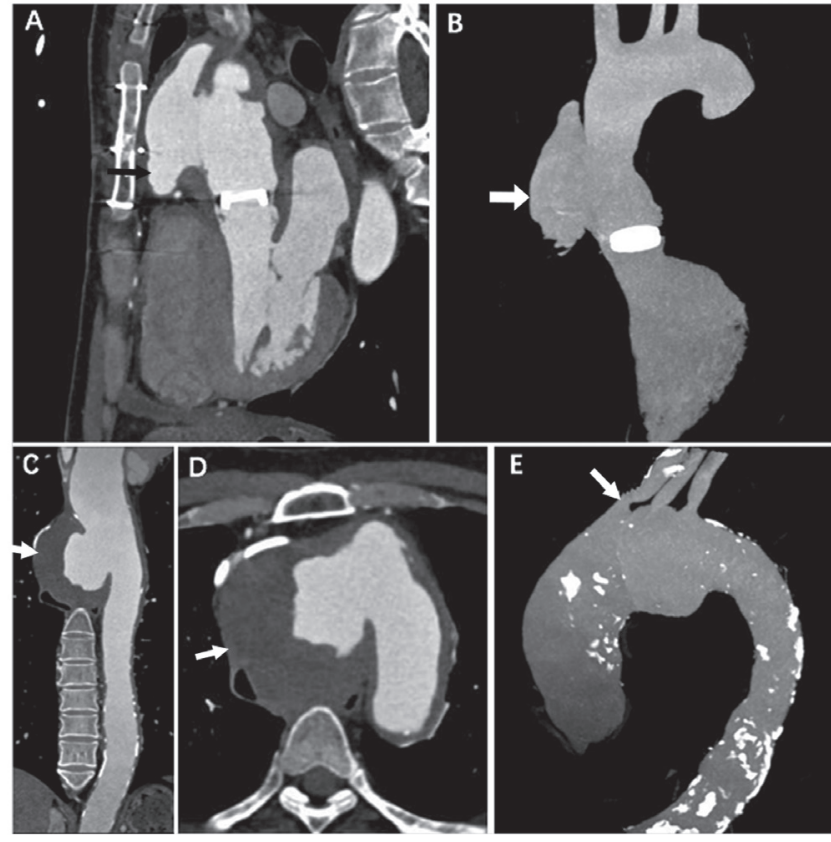

Figure 2. Patients with Behcet's disease (BD) with aneurysms in the ascending aorta. Electrocardiography-gated cardiac computed tomography (CT) scan at 9 months after a Bentall operation. (A) Curved reconstructed coronary CT image revealing large saccular pseudoaneurysms in the right wall of the ascending aorta. (B) Maximal intensity projection (MIP) image indicating no valve leakage around the artificial valve (white arrow). (C-E) Aortic CT angiographic images of a patient with vascular complications after 8 years of BD history. (C) Curved reconstructed coronary CT image and (D) axial image revealing asymmetric bulging of the right part of the aortic wall with a mural thrombus and clear boundaries (white arrow). (E) MIP image displaying an aortic arch aneurysm causing stenosis of the opening of the right brachiocephalic trunk (white arrow), left carotid artery and subclavian artery. MIP, maximum intensity projection.

unclear border of the aneurysmal wall was the only radiologic predictor associated with an elevated ESR. Vascular involvement associated with BD mainly presents as aneurysms and thrombi. In the present cohort, BD aneurysms had numerous characteristic features. The majority of BD aneurysms were located in the aorta and above the diaphragm. They were prone to having a large diameter and a homogeneously enhanced thickened wall. Thoracic aortic lesions (in the aortic sinus, ascending aorta and aortic arch) were commonly eccentric aneurysms with bulging of the right wall and were likely to be associated with mural thrombi. It may be speculated that the radiological features of vascular involvement, including inflammatory aneurysms and thrombi, are crucial features of a family of disorders associated with $\mathrm{BD}$ and may help to establish a clinical diagnosis. Pathologically, destruction of the medial layer, arterial dilatation and fibrosis are signs of active vasculitis in BD and are associated with perivascular infiltration of neutrophils, lymphocytes and plasma cells in the medial layer and adventitia, which induce fragmentation and splitting of the elastic fibers of this layer (11-13).

An accurate diagnosis of BD vasculitis is usually difficult to make only based on the initial clinical manifestations, and aneurysms are usually detected during the chronic stages of BD. In the present study, only 1 patient had massive hemoptysis and pulmonary aneurysms as initial manifestations. Pulmonary angiography revealed multiple saccular dilated pulmonary aneurysms arising from the right pulmonary artery with in situ thrombosis formation. Since BD-associated pulmonary artery occlusion is induced by in situ thrombosis, which differs from the pathogenesis of classical pulmonary thromboembolic disorders, 'pulmonary artery thrombosis' should be used for diagnosis instead of 'pulmonary emboli', and CT angiography is the best radiological tool to assess pulmonary involvement in BD (14). Cho et al (9) reported that most BD aneurysms originate from defects located in the posterior or lateral walls. However, in the present study, the most common pattern in patients with thoracic aortic aneurysms was asymmetric bulging of the right part of the aortic wall. Previous studies determined that, unlike in atheromatous aneurysms, the risk of aneurysm rupture in patients with BD was not associated with the maximum aneurysmal diameter $(15,16)$. In the present study, two patients died of an 
A

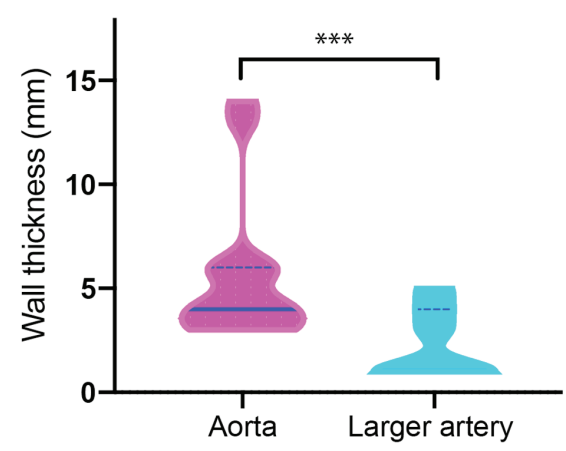

C

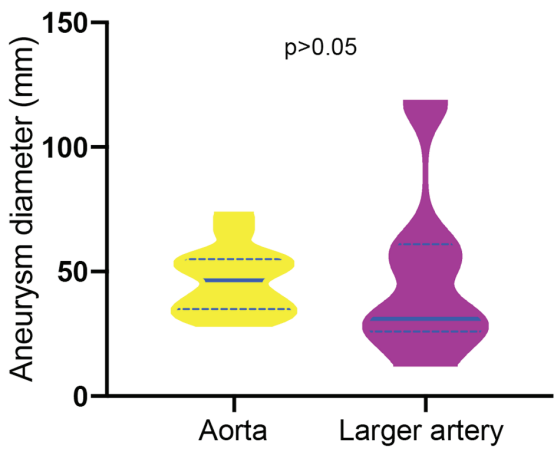

E

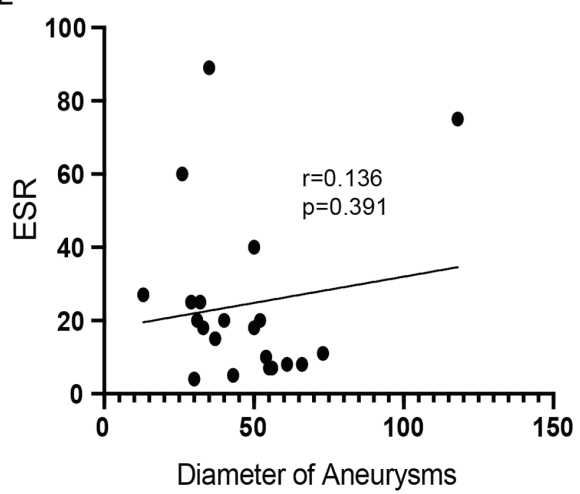

B

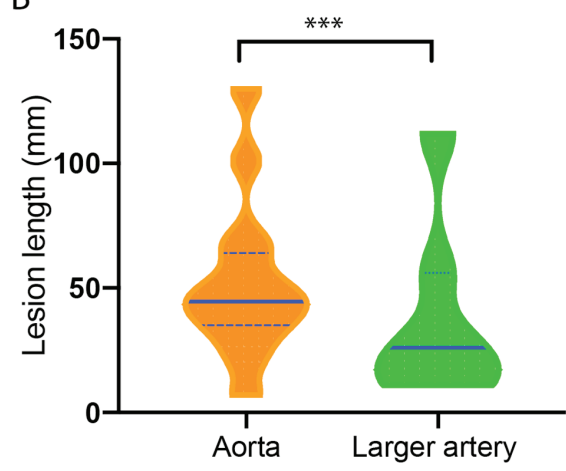

D

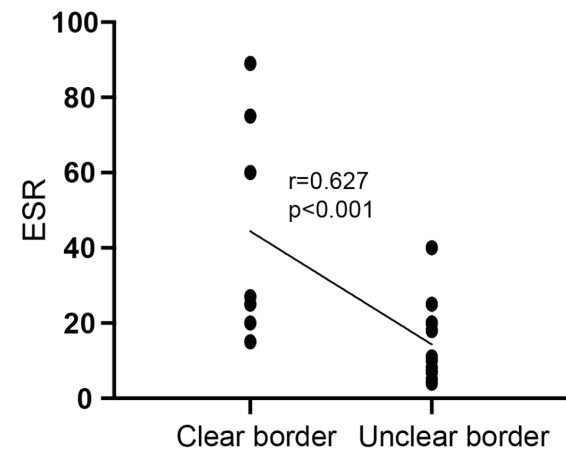

F

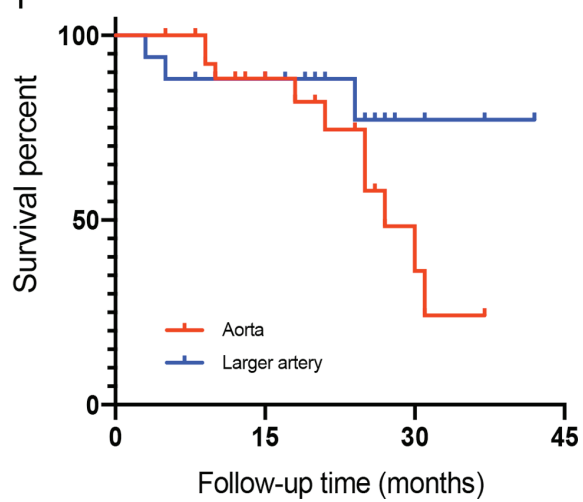

Figure 3. Comparison between groups with and without aortic and larger arterial aneurysms. Aneurysms occurring in the aorta were more likely to (A) form a thickened wall and (B) have a longer extension than those in larger arteries. ${ }^{* * *} \mathrm{P}<0.001$. (C) No significant differences in aneurysmal diameter were observed between the two groups. (D) A correlation was identified between the borders of the aneurysmal wall and the ESR. (E) The correlation between the blood erythrocyte sedimentation rate (ESR) levels and the diameter of aneurysms was not significant. (F) Kaplan-Meier survival curves for the survival outcomes of groups with and without aortic and larger arterial aneurysms. The outcome endpoints were death due to aneurysmal rupture, massive hemoptysis, recurrence after surgery, including post-operative perivalvular leakage, progression of the aneurysm and restenosis of the in-stent coronary artery. No significant difference was identified between the two survival curves (log-rank test, $\mathrm{P}=0.170$ ), but there was significant difference between the curves after 28 months of survival $(\mathrm{P}<0.01)$.

aortic aneurysm rupture and CT angiography images revealed irregular cystic changes in the thickened aortic wall. It may be speculated that cystic changes of the wall may be associated with the risk of aneurysm rupture and reflect inflammatory necrosis of the aortic wall, thus reducing pressure resistance to blood flow shocks.

In the present study, another initial feature of aneurysms associated with BD was the tendency for recurrent symptoms and involvement of multiple sites. Aneurysms may occur in various locations and simultaneously with arteriovenous thrombosis. After stent-graft implantation, recurrent pseudoaneurysms are prone to develop at the distal margins of aortic stent-grafts, and perivalvular leakage may be present after Bentall surgery. However, in larger arteries, thromboemboli are more likely to occur after stent implantation than in the aorta. The explanation for this observation may be that the stent-graft placement in actively inflamed aortic walls and continuous mechanical irritation promote pseudoaneurysm recurrence after aortic stent implantation. For larger arteries, inflammatory infiltration of the wall after stent implantation results in recurrent thromboembolism. Anastomotic and intraluminal stenosis or occlusion may result from dysfunction of the endothelium between the graft and arterial wall affected by BD (17). 

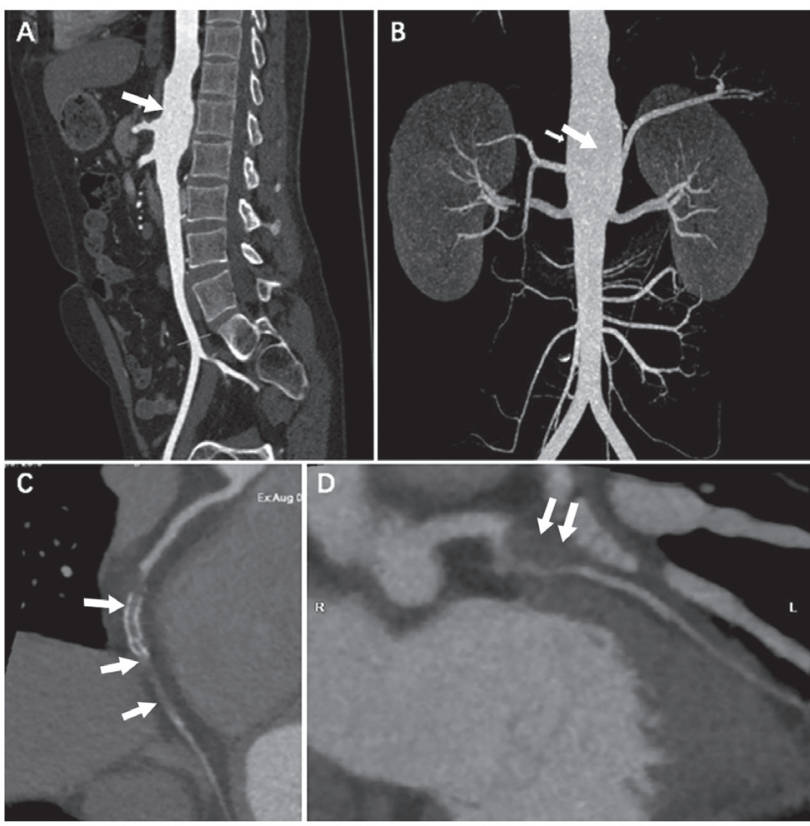

Figure 4. Patients with Behcet's disease with abdominal aortic aneurysms and coronary re-occlusion after stent implantation. (A) Coronal curved reconstructed view computed tomography (CT) image revealing a circumferentia abdominal aortic aneurysm without a thickened wall or mural thrombus formation, and no significant changes in the aneurysm were observed by ultrasound in the 6-month follow-up after medical treatment. (B) Coronal maximum intensity $\mathrm{CT}$ image reveal that no stenosis and occlusion are found in the bilateral renal arteries in that patient on $\mathrm{CT}$ angiographic image. (C) Curved reconstructed coronary CT image at the 20-month follow-up visit after right coronary stent implantation indicating a diffuse occlusion of the stent and adjacent lumen. (D) New wall thickening and severe focal stenosis of the lumen (white arrow) are found in the proximal left circumflex artery at the follow-up visit 20 months after medical treatment, which has an unclear wall boundary.

Aneurysms of BD require to be differentiated from atherosclerotic aneurysms based on the following points: i) Patient with BD aneurysm usually has a definite diagnosis and BD at a chronic stage; ii) BD aneurysms frequently feature rapid progression and have a greater risk of rupture, and consequently, huge retroperitoneal hematoma or hemoperitoneum develop as initial manifestations (9). iii) Multifocal aneurysms are usually encountered during initial manifestation of $\mathrm{BD}$, and the majority of them exhibit asymmetric bulging of the right side of the aortic wall, while concentric expansion of the aortic wall is frequently seen in atherosclerotic aneurysms.

Medical therapy with cyclophosphamide and corticosteroids has been recommended by the European League Against Rheumatism for aortic and peripheral aneurysms (18). Medical therapy with cyclophosphamide and corticosteroids is required, and monoclonal anti-TNF antibodies should be considered in refractory cases. The primary management of pulmonary artery aneurysms and thrombosis involves high-dose glucocorticoids and cyclophosphamide. BD is the only acquired disorder known to lead to the formation of pulmonary artery aneurysms, and rupture of these aneurysms may cause massive hemoptysis (19). In BD patients, refractory venous thrombosis is thought to lead to inflammation of vessel walls rather than hypercoagulability, which may result in leg ulcers that are difficult to treat.
When assessing vascular aneurysms in BD patients, arterial puncture for conventional catheter angiography should be avoided due to the possibility of new aneurysm formation at the puncture site. Compared to catheter angiography, CT angiography is non-invasive and able to provide additional information regarding the vessel walls and the presence of calcification and mural thrombi. Furthermore, characteristic $\mathrm{CT}$ imaging features of aneurysms may help to diagnose vascular involvement of BD and assess its severity, particularly in the absence of the classical clinical manifestations, which is helpful to establish a diagnosis of $\mathrm{BD}$ and to assess the efficacies of medical and surgical treatment (20).

Of note, the present study had several limitations. First, due to its retrospective nature, not all of the patients underwent $\mathrm{CT}$ angiographic examinations of the chest and abdomen. Furthermore, BD patients with vascular complications require multiple CT angiographic examination during follow-up before and after treatment, and high dosage administration of contrast agent increases the risk of contrast-induced nephropathy (21).

In conclusion, CT angiography is suitable to assess vascular complications in patients with $\mathrm{BD}$, especially of aneurysm formation. In addition, the multiplanar capability of vascular reconstruction by $\mathrm{CT}$ angiography is useful in demonstrating the manifestations of the entire spectrum of aorta and its branches in patients with $\mathrm{BD}$.

\section{Acknowledgements}

Not applicable.

\section{Funding}

This work was supported by the National Natural Science Foundation of China (grant no. 61976238), the Key Talents Training Program of Huadong Hospital (grant no.HDGG2014011), the Shanghai Hospital Development Center Program (grant no. SHDC22015025), the Health Commission of Shanghai, Wise Information Technology, Major Program of Medical Imaging (grant no. 2018ZHYL0103).

\section{Availability of data and materials}

All data generated or analyzed during this study are included in this published article.

\section{Authors' contributions}

LQ and ML made substantial contributions to the design of the research, and were involved in drafting and revising the manuscript. DBM, JFC, MW and CL prepared the figures and performed the data collection. YQH, XJ, XJG and WLW made substantial contributions to the design and data analysis for the work All authors read and approved the final manuscript.

\section{Ethics approval and consent to participate}

The institutional review board of Huadong Hospital affiliated to Fudan University (Shanghai, China) approved this retrospective study (approval no. 20160023) and written informed 
consent for MSCT examinations was obtained from each participant involved.

\section{Patient consent for publication}

This study was conducted retrospectively at one center, and the local ethical institutional review board waived the need for informed consent for this study.

\section{Competing interests}

The authors declare that they have no competing interests.

\section{References}

1. Tuzun H, Seyahi E, Arslan C, Hamuryudan V, Besirli K and Yazici $\mathrm{H}$ : Management and prognosis of nonpulmonary large arterial disease in patients with Behcet disease. J Vasc Surg 55 $157-163,2012$.

2. Uzun O, Erkan L, Akpolat I, Findik S, Atici AG and Akpolat T: Pulmonary involvement in Behcet's disease. Respiration 75: 310-321, 2008

3. Hiller N, Lieberman S, Chajek-Shaul T, Bar-Ziv J and Shaham D: Thoracic manifestations of Behcet disease at CT. Radiographics 24: 801-808, 2004.

4. Raz I, Okon E and Chajek-Shaul T: Pulmonary manifestations in Behcet's syndrome. Chest 95: 585-589, 1989.

5. Zhang SH and Zhang FX: Behcet's disease with recurrent thoracic aortic aneurysm combined with femoral artery aneurysm: A case report and literature review. J Cardiothorac Surg 12: 79, 2017.

6. Mat C, Yurdakul S, Sevim A, Özyazgan Y and Tüzün Y: Behcet's syndrome: Facts and controversies. Clin Dermatol 31: 352-361, 2013.

7. Park JH, Chung JW, Joh JH, Song SY, Shin SJ, Chung KS, Lee DY, Won JY and Kim SJ: Aortic and arterial aneurysms in behcet disease: Management with stent-grafts-initial experience. Radiology 220: 745-750, 2001

8. Reiter BP, Marin ML, Teodorescu VJ and Mitty HA: Endoluminal repair of an internal carotid artery pseudoaneurysm. J Vasc Interv Radiol 9: 245-248, 1998.

9. Cho YK, Lee W, Choi SI, Jae HJ, Chung JW and Park JH: Cardiovascular Behcet disease: The variable findings of rare complications with CT angiography and conventional angiography and its interventional management. J Comput Assist Tomogr 32: 679-689, 2008
10. Bilgin G, Sungur G and Kucukterzi V: Systemic and pulmonary screening of patients with Behcet's disease during periodic follow-up. Respir Med 107: 466-471, 2013.

11. Ishikawa S, Kawasaki A, Suzuki Y, Neya K, Wada S, Kugawa S, Hayama T and Ueda K: Progression of abdominal aortic aneurysm after endovascular stent-grafting in a patient with Behcet's disease: Report of a case. Surg Today 37: 82-85, 2007.

12. Kim SW, Lee DY, Kim MD, Won JY, Park SI, Yoon YN, Choi D and Ko YG: Outcomes of endovascular treatment for aortic pseudoaneurysm in Behcet's disease. J Vasc Surg 59: 608-614, 2014.

13. Emad Y, Abdel-Razek N, Gheita T, el-Wakd M, el-Gohary T and Samadoni A: Multislice CT pulmonary findings in Behcet's disease (report of 16 cases). Clin Rheumatol 26: 879-884, 2007.

14. Zhang X, Dai H, Ma Z, Yang Y and Liu Y: Pulmonary involvement in patients with Behcet's disease: Report of 15 cases. Clin Respir J 9: 414-442, 2015.

15. Seyahi E: Behcet's disease: How to diagnose and treat vascular involvement. Best Pract Res Clin Rheumatol 30: 279-295, 2016.

16. Goudard Y, Pierret C, de La Villéon B, Mlynski A and de Kerangal X: In situ repair of a primary Brucella-infected abdominal aortic aneurysm: Long-term follow-up. Ann Vasc Sur 27: 241.e1-e5, 2013.

17. Tuzun H, Besirli K, Sayin A, Vural FS, Hamuryudan V, Hizli N, Yurdakul S and Yazici H: Management of aneurysms in Behcet's syndrome: An analysis of 24 patients. Surgery 121: 150-156, 1997.

18. Hatemi G, Christensen R, Bang D, Bodaghi B, Celik AF, Fortune F, Gaudric J, Gul A, Kötter I, Leccese P, et al: 2018 update of the EULAR recommendations for the management of Behcet's syndrome. Ann Rheum Dis 77: 808-818, 2018.

19. Ceylan N, Bayraktaroglu S, Erturk SM, Savas R and Alper H: Pulmonary and vascular manifestations of Behcet disease: Imaging findings. AJR Am J Roentgenol 194: W158-W164, 2010.

20. Alkaabi JK and Pathare A: Pattern and outcome of vascular involvement of Omani patients with Behcet's disease. Rheumatol Int 31: 731-735, 2011.

21. Albrecht MH, Bickford MW, Nance JW Jr, Zhang L, De Cecco CN, Wichmann JL, Vogl TJ and Schoepf UJ: State-of-the-art pulmonary CT angiography for acute pulmonary embolism. AJR Am J Roentgenol 208: 495-504, 2017. 